\title{
Neural Network and Tree Search Algorithms for the Generation of Path-Following (Trail-Making) Tests
}

\author{
Michael D. Lee, Mark Brown and Douglas Vickers" \\ Department of Psychology, University of Adelaide \\ Adelaide, South Australia 5005 \\ Tel: +61883035662 \\ Fax: +61883033770 \\ E-mail: psyd-vic@arts.adelaide.edu.au
}

\begin{abstract}
Due to their sensitivity to cognitive impairment, simplicity of administration and low cost, path-following tests, such as the HalsteadReitan Trail Making Test (TMT) and the Zahlen-Verbindungs-Test (ZVT), are among the most frequently used neuropsychological tests in the assessment of brain damage. Despite their usefulness, however, such tests have been constructed in an unprincipled manner which does not facilitate repeated testing, systematic investigation or theoretical interpretation. The reason for their unprincipled development is that the selective generation of the required pathways constitutes a computational problem that is NPcomplete. This paper outlines two practical solutions to the problem of generating ZVT matrices. The first employs a Hopfield-style neural network and the second employs a heuristic-driven tree search. The characteristics of the pathways generated by these two solutions are compared, and ways of manipulating the relative difficulty of alternative test forms are discussed.
\end{abstract}

\section{KEYWORDS}

Zahlen-Verbindungs-Test, neural networks, tree-search, path-following, neuropsychology, optimization

"To whom correspondence should be addressed. 


\section{INTRODUCTION}

Since the beginning of this century, various forms of visually guided path-finding and path-following tasks have been used in experimental and clinical work with human subjects. The first published path-finding task appears to have been a series of maze tests, developed by Porteus in 1913, the most recent forms of which still resemble the earliest version (Porteus, 1965). Similar forms of maze tests include Gibson's Spiral Maze (Gibson, 1964; 1965), the Austin Maze Test (Walsh, 1985), the Elithorn Perceptual Maze Test (Elithorn, 1955; Elithorn, Jones, Kerr, \& Lee, 1964; Elithorn, Kerr, \& Mott, 1960), and the Group Maze Test (Nevo, Arronson, \& Israeli, 1984). More particularly, one group of maze-like path-following tasks, which have been popular with both clinicians and experimenters, are the socalled trail-making tests. Various forms of these tests have been produced, dating back from a prototype known as the Taylor Number Series - a test which required the subject to connect a series of numbers 'scattered randomly' on a sheet of paper (Brown, Casey, Fisch, \& Neuringer, 1958). These include the Partington Pathways Test (Brown et al,, 1958), the Tsai Number-Joining Test (Tsai, 1969), and the Tsai-Partington Numbers Test (Ammons, 1955). The most widely used version of such tests, known as the Trail Making Test (TMT), was initially incorporated as a performance subtest in the Army Individual Test of General Ability, and later added to the Halstead-Reitan Neuropsychological Battery (Reitan, 1955; 1958; 1992; Reitan \& Wolfson, 1988). Meanwhile, one of the most recent of such tests is the so-called Zahlen-Verbindungs-Test (ZVT), or Number-Joining-Test, developed in Germany by Oswald and Roth (1978; 1987).

Because they are simple, inexpensive, and quick to administer, such path-following tests are particularly advantageous for clinical applications. For example, the TMT is perhaps the most frequently employed neuropsychological test ever developed (Butler, Retzlaff, \& Vanderploeg, 1991). It is also widely regarded as one of the most sensitive indicators of brain damage (Corrigan \& Hinkeldey, 1987; Crawford, Parker, \& McKinlay, 1992; Lezak, 1995; Spreen \& Strauss, 1991). Meanwhile, various forms of the ZVT have consistently shown moderate to high correlations with psychometric measures of intelligence (Oswald \& Roth, 1978; 1987; Vernon, 1993; Vernon \& Weese, 1993), and have found a variety of clinical applications in the fields of intelligence, aging, psychopharmacology and gerontology (Neubauer, 1993; Oswald \& Fleischmann, 1985). At the same 
time, like their predecessors, both tests suffer from two major disadvantages. The root problem is that neither test appears to have been constructed in accordance with a set of clearly specifiable principles capable of implementation by an algorithm which could generate other, theoretically or measurably equivalent test forms. As pointed out by Geffen (1995), in a more general critique of approaches to neuropsychological assessment, one consequence of this is that there has been no systematic, theoreticallydriven, experimental study of path-following performance, and little progress has been made in theorizing about an obviously fundamental ability. A second consequence is that no procedure for generating equivalent but different test forms has been devised, with the result that an insufficient number of alternative test forms are available for instruction, experimentation or repeated testing of performance (although a truncated form of the TMT designed for children has been published by Reitan \& Wolfson, 1992, while two alternative, shorter (5x6) ZVT test forms have been presented by Oswald \& Fleischmann, 1995).

The purpose of the present paper is to examine the reason for this situation and to present two practical solutions, capable of generating unlimited numbers of ZVT test forms, from which a selection possessing various desired characteristics can then be made. We begin with a brief consideration of the TMT. While this appears to offer more scope for theoretical development, we argue that the ZVT lends itself more readily to the generation of multiple distinguishable test forms. We then outline two procedures by which this may be achieved, and conclude with some suggestions for ways in which the relative difficulty of alternative forms may be measured and manipulated.

\section{THE TRAIL MAKING TEST}

The TMT consists of two parts. In Part A, circles, numbered from 1 through 25 , are 'randomly distributed' on a page. Subjects have to connect the circles, as quickly as possible, by drawing a continuous line, or 'trail', from the circle numbered 1 up to that numbered 25. Part B is similar, except that the 25 circles contain numbers or letters, and must be connected in the order 1, A, 2, B,..., L, 13. Errors must be corrected before continuing. For both parts $A$ and $B$ the score is the total time needed to correctly complete the task. 
As Vickers, Vincent and Medvedev (in press) point out, the above customary description of the TMT is somewhat misleading, since the pathways in both forms A and B are obviously constrained, and take the form of spiral, self-avoiding curves, so that it is possible to link up the circles with a continuous line that does not intersect itself. The self-avoiding constraint was presumably applied to facilitate the task of monitoring and scoring the subject's performance. However, the spiral constraint arises because the problem of sequentially assigning numbers and letters to a set of randomly distributed circles (or nodes) in such a way that the resulting pathway would be self-avoiding would potentially involve considering all possible links between all possible circles. The combinatorial explosion involved in considering $n$ ! possible itineraries is generally recognized as computationally intractable for any but small numbers of $n$ (Lawler, 1985). For this reason, it seems likely that paths A and B were not generated in this way, but were probably constructed by first drawing 'random-looking', but self-avoiding, curves, and then sequentially assigning the numbers or letters to successive inflection points on these curves. In other words, the immediate motivation underlying the paths was not to select one out of an indefinite number of pathways linking a set of 'randomly distributed' nodes, but to fit an irregularly segmented curve into a confined space in such a way that the curve was evenly distributed within the space. As evidenced by children's drawings and by 'rope-coiling' exercises, such as mazes and labyrinths, it is extremely difficult by freehand methods to distribute such a curve fairly evenly over a rectangular surface, and still preserve some local randomness, without conforming to some familiar overall configuration, such as a spiral or boustrophedon structure (Bord, 1976; Fein, 1993; Matthews, 1922/1970).

As Vickers et al. (in press) go on to argue, such curves are reminiscent of so-called 'space-filling' curves which, in the limit, pass through every point in the plane, and are more like two- dimensional objects than onedimensional lines. Consistent with this, paths $A$ and $B$ were found by Vickers et al. to have box-covering dimensions of 1.207 and 1.225 , respectively (Voss, 1988). Thus these paths resemble fractal curves, which are produced by recursive processes in which the results of applying a set of geometrical transformations to a 'seed' element are fed back in as input to the transforming process (Mandelbrot, 1982). Using a tiling of the transformations suggested by Peitgen, Jürgens and Saupe (1992) as an example, Vickers et al. argue that TMT pathways may be generated in a 
similar manner. Since Barnsley's (1988a, b) work on iterated function systems has shown that naturalistic visual images can be encoded in compressed form by the parameters of a collage of affine transformations, applied to a number of reduced copies of a seed element, this means that any TMT pathway - no matter how complex - can be encoded in this way. Conversely, such an iterated function system provides a principled procedure for the generation and systematic variation of pathways of any desired degree of complexity. For example, path structure can be manipulated by varying the parameters and complexity of the seed element, by changing the number, parameters and configuration of the transformations, or by any combination of these variations. This in turn suggests theoretically oriented questions, such as whether performance is sensitive to invariants across the set of geometric transformations employed.

At the same time, the constraint that pathways be self-avoiding in the TMT means that it is not obvious with this method how to produce large numbers of self-avoiding pathways which are equivalent in complexity and yet stochastically different. The self-avoiding constraint seems necessary because there is no other constraint on the lengths of component line segments and an entirely random distribution and interconnection of nodes would make the task confusing for the subject and difficult - or impossible for the experimenter to monitor for errors. As a result, the generation process is restricted to using tilings of recursive transformations in which the component line segments are contiguous only at the end points. This means that, while it is easy to manipulate the qualitative characteristics of alternative pathways (for example, by employing an asymmetrical rather than a symmetrical seed element, or by changing the tiling), it is difficult to produce large numbers of self-avoiding pathways which are equivalent in complexity and yet stochastically different. Since multiple alternative pathways are required for repeated testing and instruction, we have therefore focussed on the ZVT, in which the distance covered in successive path segments is restricted to one cell (vertically, horizontally or diagonally), but in which there are no obvious constraints on self-intersections.

\section{THE ZAHLEN-VERBINDUNGS-TEST}

The original ZVT involves a 9x10 matrix of circled numerals from 1 to 90 , which are positioned irregularly, with the sole explicit constraint that 
successive integers occupy immediately adjacent circles (vertically, horizontally or diagonally), thus forming a path through the matrix. A well known and long standing problem with the same structure is the so-called Knight's Tour, a chess puzzle in which the problem is to find a path which will allow the Knight to traverse the entire board, landing exactly once on each square (Falkener, 1892; Murray, 1913). In fact, for an $8 \times 8$ matrix the problem of generating a pathway for the ZVT is equivalent to that of specifying a non-trivial (irregular) King's Tour, since the next number must always be located (one King's move away) in an immediately adjacent circle.

Employed as a clinical tool, the ZVT requires a subject to draw a path correctly connecting the integers in their natural order, with the score being the time to accomplish this. Errors must be corrected immediately by the subject or noted by the experimenter. In recent studies by Vernon (1993), the subject has been required to connect up the numbers in the reverse order, or, as in part B of the TMT, to connect up some combinations of letters and numbers. However, the standard ZVT consists of four test forms, A, B, C, and $D$, using naturally connected numbers only, and with test $D$ being a mirror image of $B$.

Although there is no obvious limitation on the number of selfintersections in the case of the ZVT, the matrices do appear to have been constructed using implicit constraints, which are not clearly evident even from a perusal of the completed structure. However, if we plot the cumulative record of traversed cells at successive intervals of 15 digits, as in Figure 1, then the constraints become obvious. In matrix A, for example, the overall progression follows a clockwise spiral 3-4 circles thick, traversing all the outer cells before approaching the center. In Matrix B, the overall progression begins as clockwise before twisting and reversing. Matrix $\mathrm{C}$ may appear a little more complex, but actually follows a quite regular, boustrophedon progression. Moreover, the pathways in alternative test forms differ with respect to a number of descriptors, such as number of selfintersections and number of continuous line segments.

As with the TMT, the reason for the unprincipled construction of the ZVT matrices is the combinatorial explosion of the number of alternatives which must be considered in order to find valid paths as the number of circles or cells in the matrix increases. As is also the case with the TMT, however, it is possible to devise practical solutions to the construction of ZVT matrices. One early manual solution, involving the use of a heuristic 
a

d

S100000000 100000000 000000000 0000000000

0000000000

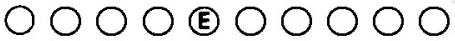

0000000000

0000000000

0000000000

b

$\theta$

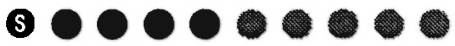

s000000000 ०००००००००0 0000000000 0000000000 - 0000000 - 00 O E 0000

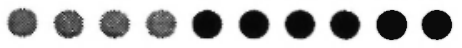

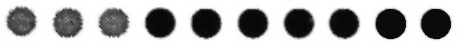
-

C

f

s000000000 -

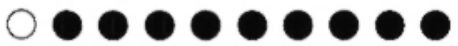
00000000 00000000 0000 트은? 000000000 . 000000000 00000000 .

s000000000 - 00000000 - 00000000 - 00000 00000000 0000000 000000000 0000000000 0000000000

Fig. 1: Cumulative record of cells traversed in test form A of the ZVT. 
method, has been suggested by Pearce and Vickers (in press). The following two solutions have the advantage of being realized in computer implementations, and of being capable of generating unlimited numbers of alternative ZVT matrices in a negligible amount of time.

\section{A NEURAL NETWORK SOLUTION}

The first solution is a direct adaptation of the neural network described by Mehta and Fulop (1993) for the generation of Hamiltonian paths. Clearly, the notion of a Hamiltonian path, which passes through each of a set of arbitrarily interconnected points or cells exactly once, corresponds exactly to the notion of a valid ZVT path.

The problem of finding Hamiltonian paths is, in turn, related to the notorious 'Traveling Salesperson' problem, which can be posed as follows: Given a set of $n$ cities, and a specified cost incurred in moving between any two of them, devise an itinerary such that (a) each city is visited exactly once and (b) the total cost of the itinerary is a minimum. Indeed, the only twc important differences between these 'NP-complete' problems (Goldschlager \& Lister, 1988) are the following: Firstly, whilst it is possible to move between any arbitrary pair of cities in the Traveling Salesperson problem, only movement between connected points is allowed in the Hamiltonian path problem. Secondly, the Traveling Salesperson problem involves the optimization of valid path solutions, in terms of minimizing a cost function; in the Hamiltonian path problem, each valid path constitutes an equally good solution. Thus, the Hamiltonian path problem can be viewed as a Traveling Salesperson problem in which the cost of traveling between nonconnected points is infinite, and no requirement is made that the total cost of a valid path be a minimum.

Mehta and Fulop's (1993) network exploits this relationship by extending and refining a Hopfield- style network (Hopfield \& Tank, 1985) which was designed to solve Traveling Salesperson problems. As detailed in Table 1, Mehta and Fulop (1993) report that their network is able to find Hamiltonian paths in a reasonable amount of time for problems with up to 500 points, given sufficiently high levels of connectedness between the points. Recalling the previously noted correspondence between cells of pathfollowing matrices and points in Hamiltonian paths, it seems clear that 500 
Table 1:

Success rates of Mehta and Fulop's Network.

Based on Mehta and Fulop (1993), Table 1.

\begin{tabular}{|c|c|c|c|c|c|c|}
\cline { 3 - 7 } \multicolumn{2}{c|}{} & \multicolumn{5}{c|}{ Connectivity } \\
\cline { 3 - 7 } \multicolumn{1}{c|}{} & $\mathbf{1 0}$ & .15 &. $\mathbf{2 0}$ &. $\mathbf{5 0}$ &. $\mathbf{9 0}$ \\
\cline { 2 - 7 } & $\mathbf{2 0}$ & $20 \%$ & $20 \%$ & $100 \%$ & $100 \%$ & $100 \%$ \\
\cline { 2 - 7 } & $\mathbf{5 0}$ & $20 \%$ & $100 \%$ & $100 \%$ & $100 \%$ & $100 \%$ \\
\cline { 2 - 7 } & $\mathbf{1 0 0}$ & $30 \%$ & $100 \%$ & $100 \%$ & $100 \%$ & $100 \%$ \\
\cline { 2 - 7 } $\begin{array}{c}\text { Number } \\
\text { Points }\end{array}$ & $\mathbf{2 0 0}$ & $\mathbf{5 0} \%$ & $100 \%$ & $100 \%$ & $100 \%$ & $100 \%$ \\
\cline { 2 - 7 } & $\mathbf{3 0 0}$ & $100 \%$ & $100 \%$ & $100 \%$ & $100 \%$ & $100 \%$ \\
\cline { 2 - 7 } & $\mathbf{4 0 0}$ & $100 \%$ & $100 \%$ & $100 \%$ & $100 \%$ & $100 \%$ \\
\cline { 2 - 7 } & $\mathbf{5 0 0}$ & $100 \%$ & $100 \%$ & $100 \%$ & $100 \%$ & $100 \%$ \\
\hline
\end{tabular}

points is more than adequate. The ZVT uses $9 \times 10$ matrices and hence requires Hamiltonian paths to be generated through only 90 points.

Of more concern is the deterioration in the success rate of Mehta and Fulop's network when the points are sparsely interconnected. For a general $n \times m$ matrix (where $n, m \geq 2$ ), the connectivity can be calculated according to the formula:

$$
\text { connectivity }=\frac{8 n m-6 n-6 m+4}{(n m)^{2}}
$$

Thus the connectivity of a $9 \times 10$ ZVT matrix can be calculated to be 0.075 ( $3 \mathrm{dp}$.). According to Table 1, this means that Mehta and Fulop's network would be able to generate ZVT matrices successfully less than $20 \%$ of the time, if at all. Indeed, our simulations for a $9 \times 10$ matrix failed to generate a single solution after 100 separate attempts. There is, however, no principled reason why the ZVT matrices should be $9 \times 10$, and so it would seem reasonable for present purposes to use Mehta and Fulop's network to generate path-following matrices of the largest size of which the network is capable. Fortunately, according to Eq. (1), 8x8 ZVT matrices have a connectivity value of 0.103 ( $3 \mathrm{dp}$.), which, as can again be seen from Table 1 , is just large enough to generate solutions with a $20 \%$ success rate. 
Accordingly, we now summarize the definition, operation and interpretation of Mehta and Fulop's (1993) network as we have applied it to the problem of generating $8 \times 8 \mathrm{ZVT}$ path-following test matrices.

\subsection{Definition of the Network}

The network consists of a $64 \times 64$ square grid of 4,096 nodes, each of which has an activation value, $A$, capable of taking values in the interval $[0,1]$, but which are all initialized to the value 0.5 . Two nodes in the grid, say $(x, i)$ and $(y, j)$, have a fixed connection weight, $w$, given by:

$$
\begin{aligned}
& w_{x i, y i}=-H \delta_{x, y}\left(1-\delta_{l, j}\right)-V \delta_{i, i}\left(1-\delta_{\mathrm{x}, \mathrm{y}}\right)- \\
& S \delta_{x, y} \delta_{i, j}-D c_{x y}\left(\delta_{i, j+1}+\delta_{i, j-1}\right)
\end{aligned}
$$

where $H=0.7, V=0.7, S=0.6, D=0.15, \delta_{i, j}$ is the Kronecker delta function, and $c_{x y}$ is a measure of the cost of moving from cell $\mathrm{x}$ to cell $\mathrm{y}$ in the ZVT matrix.

These cost values are defined in the following way: if the cells $x$ and $y$ are adjacent in the matrix, the cost is a small random number chosen from a uniform distribution across the interval [0,0.05]. If cells $x$ and $y$ are not adjacent, the cost of moving between them is set to the much larger value of 1. The rationale behind this method of selecting cost values is clear if the Hamiltonian path ZVT problem is viewed as a special case of the Traveling Salesperson problem, as described earlier. Moves between adjacent cells are encouraged by the small costs assigned to moving between them, whilst the costs involved in making an invalid move to a non-adjacent cell are prohibitively large. Different Hamiltonian paths, corresponding to different ZVT matrices, can be generated by altering the seed value of the random number generator which assigns the small cost measures.

It is worth noting that this pattern of costs involved in translating the ZVT problem into a Traveling Salesperson problem prohibits the use of the well-known 'elastic net' optimization networks (Durbin \& Willshaw, 1987; Fritzke \& Wilke, 1991). These elastic nets require that the cities can be arranged on a plane (typically a two dimensional Euclidean plane) in such a way that the distance between any two cities corresponds to the cost of moving between those cities. It takes only a little thought to realize that the patterns of costs between ZVT matrix cells we have described above cannot be represented by the distances between a planar arrangement of the cells. In 
fact, the cells cannot be appropriately embedded in any metric space whatsoever. To see this, consider a central cell and the two cells which flank it immediately to the left and right, respectively. In accordance with the assumed distribution of costs, the central cell must be within 0.05 of both of the flanking cells, since they are adjacent. The two flanking cells, however, are not adjacent, and must therefore be separated by a distance of 1 . Since these three distances do not satisfy the triangle inequality, an appropriate geometric configuration for the three cells cannot be found in any metric space (Shepard, 1974).

\subsection{Operation of the Network}

On each iteration of the network, the activation of the node at $(x, l)$ in the grid is determined by first calculating an input value, $a$, given by:

$$
a_{x, i}=\lambda\left(\sum_{y=1}^{\hat{0}} \sum_{j=1}^{\hat{\mathrm{o}}}\left(A_{y j} w_{y j, x i}\right)+1\right)
$$

and then passing this value through a bounded-linear function, resulting in a final activation of $A$, given by:

$$
A_{x i}= \begin{cases}0 & \text { if } a_{x i}<-0.5 \\ 0.5+a_{x i} & \text { if }-0.5 \leq a_{x i} \leq 0.5 \\ 1 & \text { if } a_{x i}>0.5\end{cases}
$$

The value $\lambda$ in Eq. (3) takes the initial value 0, and is increased by a value of 0.01 each time successive iterations result in each node's activation value changing by less than a tolerance level of 0.2 .

This iterative process is terminated when, on two successive values of $\lambda$, all cell activation values are unchanged, and the network grid has property that each row and each column has one node with an activation value of 1 , and all other nodes have activation values of 0 . When these criteria are satisfied, the network is said to be in a stable state.

\subsection{Interpretation of the Network}

A stable state of the network potentially defines a ZVT matrix solution, which can be evaluated by interpreting rows of the grid as corresponding to cells in the path-following matrix. If the node $(x, j)$ in the grid is active in the 
stable state, then the path solution passes through the cell associated with row $x$ on its $j^{\text {th }}$ move. An ordered sequence of cells which constitutes an entire path solution, therefore, can be generated by considering the columns of the stable matrix in sequence.

This process is graphically depicted in Figure 2 for the case of a $3 \times 3$ path-following matrix and its associated $9 \times 9$ network. The rows of the network are identified with cells in the matrix, labelled ' $A$ ' through ' $\mathrm{T}$ ', as shown. The 9 active cells in the stable state of the network are shaded, and define the path solution which is also shown. An example of the final pathway for an $8 \times 8$ matrix generated in this way is given in Figure 3.

It is possible that the ZVT path defined by the stable state may not be a valid one. In particular, it sometimes happens that a lone 'jump' to a nonadjacent cell in the matrix is inserted in an otherwise valid path. We employed a simple algorithm to evaluate each potential path solution to verify whether or not it was a complete solution. Of the 600 random number seeds we tried, the network generated valid ZVT matrices on 64 occasions, a success rate closer to $10 \%$ than the $20 \%$ that was expected from Table 1 .

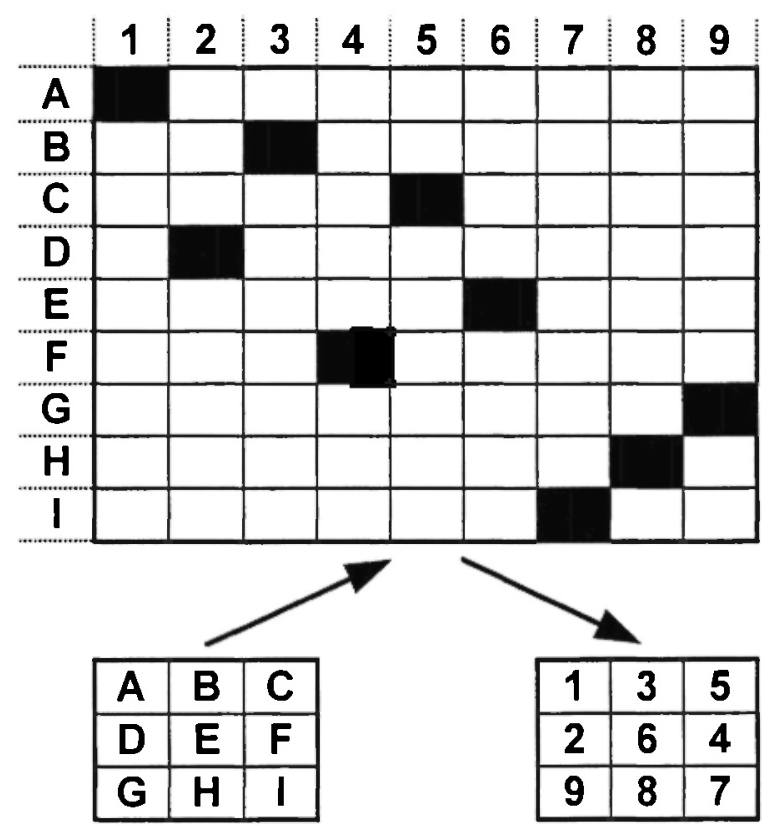

Fig. 2: Interpreting the stable state of the neural network as a path solution. 


\begin{tabular}{|c|c|c|c|c|c|c|c|}
\hline 49 & 50 & 47 & 46 & 20 & 22 & 23 & 24 \\
\hline 51 & 48 & 45 & 17 & 21 & 19 & 27 & 25 \\
\hline 52 & 54 & 16 & 44 & 18 & 42 & 26 & 28 \\
\hline 53 & 55 & 14 & 15 & 43 & 41 & 29 & 31 \\
\hline 11 & 13 & 56 & 58 & 40 & 38 & 32 & 30 \\
\hline 12 & 10 & 57 & 1 & 59 & 39 & 37 & 33 \\
\hline 9 & 7 & 4 & 60 & 2 & 64 & 36 & 34 \\
\hline 8 & 5 & 6 & 3 & 61 & 62 & 63 & 35 \\
\hline
\end{tabular}

Fig. 3: An example of a completed $8 \times 8 \mathrm{ZVT}$ matrix.

The average computational time taken to generate one of these solutions was approximately 15 minutes, on a Sun SS10 Model 41.

\section{A TREE SEARCH SOLUTION}

Our second solution to the problem of generating ZVT matrices takes the form of a heuristic-driven tree search, of a type characteristic of the field of Artificial Intelligence in the 1970s (Newell \& Simon, 1972). The essence of this general approach is that the necessity to consider an unmanageably large number of alternatives at any stage in the search for a solution can be alleviated by devising some heuristic procedure by which appropriate decisions can be made in a reasonable amount of computational time. Applying this principle to the generation of ZVT matrices involves specifying heuristics which suggest individual path moves that are likely to constitute a valid solution. Such heuristics effectively 'prune' the search tree of potential path moves, removing those which will prevent a complete Hamiltonian path through the ZVT matrix from being generated.

The heuristic we employed is founded on the notion of 'constrictions', which may be defined as a pattern of filled cells with a path of empty cells one cell wide leading from one area of unused cells to another. Figure 4 shows two types of constrictions which, by virtue of the axes conferred by the matrix boundaries, impose different constraints on path generation. 


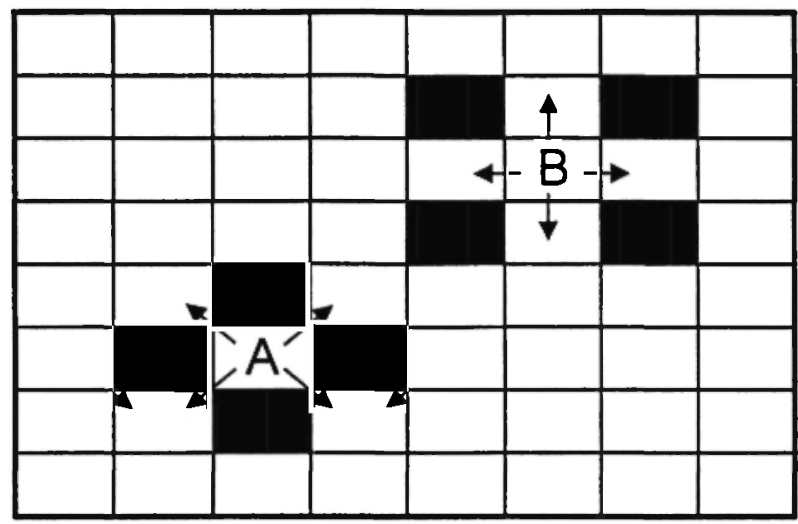

Fig. 4: The two basic constriction patterns.

With either type, it can be seen that, in terms of its potential to form a valid solution, a move through a constriction is something of a defining moment. In the first place, if a path passes through a constriction, and there is no available passage through the wall thus formed, an isolated region will be created which prevents the construction of a solution. Secondly, moving through a constriction may create two configurations in the matrix, which we term 'dead-ends', both of which can be entered but not exited. Any partially generated path solution may contain one such dead-end (the last path move necessarily fills a dead-end), but the creation of two dead-ends renders any prospective path untenable.

The number of dead-ends formed by the creation of a constriction is evaluated using a technique of coloring the cells in the matrix with numbers. Cells are colored using a depth-first recursive algorithm, with an initial color value of one which is incremented by the entry into a potential constriction. That is, from the cell currently being considered as a path move, a randomly selected neighbor is colored. Next, one of its available neighbors is colored, and so on, until a cell is encountered which has no available neighbors. At this point, we retrace our steps to the most recently considered cell which still has an available neighbor, proceed to color this neighbor, and continue as before.

This process will terminate when all available cells which can be reached from the path move we are contemplating have been assigned a coloring number. Furthermore, the coloring process generates a record, for each cell that is colored, of the minimum color assigned to the neighbors of that cell. 
If none of the cells colored after the passage through a constriction has a neighbor with a smaller color, we conclude that the constriction does create a dead-end.

For example, in Figure 5, a move is being considered from cell ' 15 ' to its neighbor on the right. This cell is assigned the number 1 (the numbers in the non-shaded cells are color numbers), and the remaining unvisited cells in the matrix are similarly 'colored' by a depth first recursive algorithm which increments the color number by one on the passage through a constriction. The fact that the color 4 below cell ' 15 ' encounters a neighboring cell colored 1 results in no dead end being counted, and the number of colored cells establishes that the move will not establish an isolated area in the matrix. Note that the exact results of any given coloring process depend on the random selection of unvisited neighboring cells, and that the final coloring shown here is therefore only one of many possibilities, although all of these possibilities will produce the same final dead-end count and isolation check.

\begin{tabular}{|c|c|c|c|c|c|c|c|}
\hline 3 & 3 & 14 & 15 & 1 & 1 & 2 & 2 \\
\hline 3 & 3 & 13 & 4 & 11 & 2 & 2 & 2 \\
\hline 3 & 3 & 4 & 12 & 2 & 10 & 2 & 2 \\
\hline 3 & 4 & 4 & 2 & 9 & 2 & 2 & 2 \\
\hline 3 & 3 & 5 & 2 & 8 & 2 & 2 & 2 \\
\hline 3 & 2 & 2 & 6 & 7 & 2 & 2 & 2 \\
\hline 1 & 3 & 2 & 2 & 2 & 2 & 2 & 2 \\
\hline 2 & 2 & 2 & 2 & 2 & 2 & 2 & 2 \\
\hline
\end{tabular}

Fig. 5: The coloring process.

Figures 6 and 7 depict scenarios involving isolation and multiple deadends, respectively. In Figure 6, the path move being considered is from cell ' 19 ' to the cell labelled '?', which involves the passage into a constriction, and requires the consideration of the dead-end above the further constriction at cell ' $\mathrm{B}$ '. This dead-end will be identified by the coloring process at both 


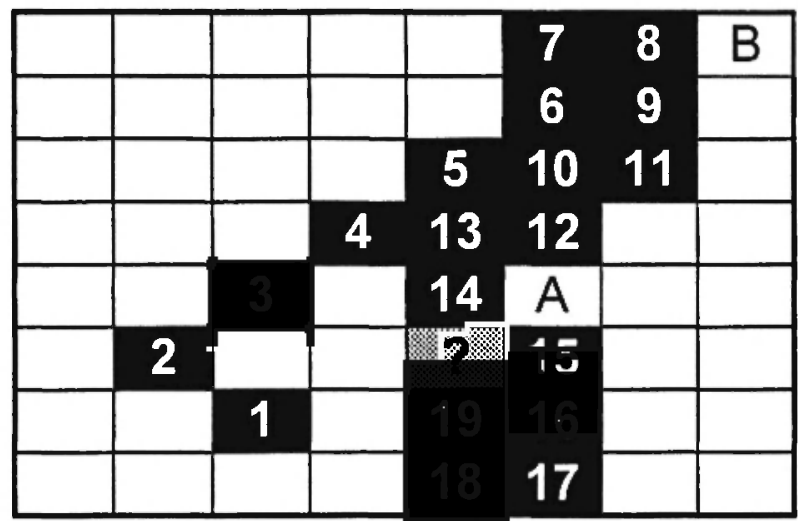

Fig. 6: A path move requiring rejection on the grounds of isolation.

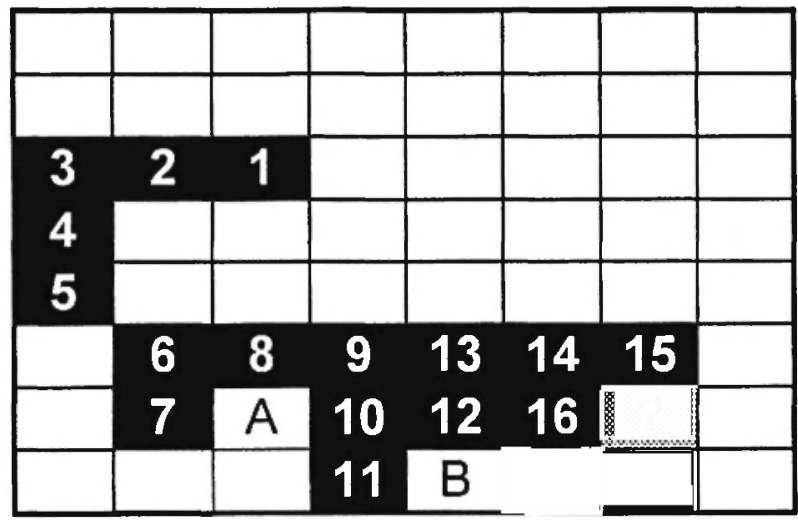

Fig. 7: A path move requiring rejection on the grounds of multiple deadends.

constriction cells ' $A$ ' and ' $B$ '. To avoid an inappropriate double-counting of this dead-end, therefore, only its identification at cell ' $B$ ' is counted. Thus, the potential path move to the cell labelled '?' is rejected solely on the grounds that it creates an impermeable barrier partitioning the matrix into two isolated regions of unvisited cells.

In Figure 7, the path move being considered is the one from cell ' 16 ' to the cell labelled '?'. This move represents the passage through a constriction, and the resulting search for dead-ends will establish two at the constriction cells labelled ' $A$ ' and ' $B$ '. Note that the coloring process will 
reach every unvisited cell in the matrix, and that, therefore, the move being contemplated is to be rejected solely on the basis that it creates more than one dead-end.

In summary, the basis of the tree search algorithm is to continue to move from the currently occupied cell to a randomly selected, unvisited, adjacent cell, unless such a move involves a constriction. If the move creates a constriction, we accept the move only if the newly created constriction leads to at most one dead-end. If the move constitutes an entry into an existing constriction, we accept the move only if every unvisited cell in the matrix can still be reached. A valid path-following solution is attained when every cell in the matrix has been visited.

In terms of the efficiency of generating solutions, this tree search strategy represents a significant improvement over the neural network approach. For $8 \times 8$ matrices, virtually every starting seed will result in a valid path, and the average computational time to generate such paths is of the order of one tenth of a second, on a Sun SS10 Model 41. Furthermore, the tree search algorithm is less affected by the required dimensions of the ZVT matrix than is the neural network. Only when matrices of the order of $15 \times 15$ are considered does the approach begin to fail to generate solutions for some starting seeds, and not until 20×20 matrices are considered does this failure become prohibitively common. If, however, a valid solution is to be generated for a given seed, the time taken to produce that solution is, for practical purposes, not affected by the dimensions of the matrix, and remains effectively instantaneous.

We suspect that this deterioration is caused by the increased likelihood, in these larger matrices, of scenarios such as the one we have termed 'looping' which often prevent the generation of a valid solution, but which are not circumvented by the application of our heuristics. For example, in Figure 8, the path move to cell '?' being considered does not form isolated regions of unvisited cells in the matrix, nor does it involve consideration of multiple dead-ends. However, a move to cell '?', does mean that the path will have to visit every cell in the semi-enclosed region it has entered, before it exits through either of the constriction cells labelled ' $A$ ' and ' $B$ '. Given the random nature of the movement within this semi-isolated region, which is otherwise devoid of constrictions, the likelihood that the path will visit each cell before exiting is small. 


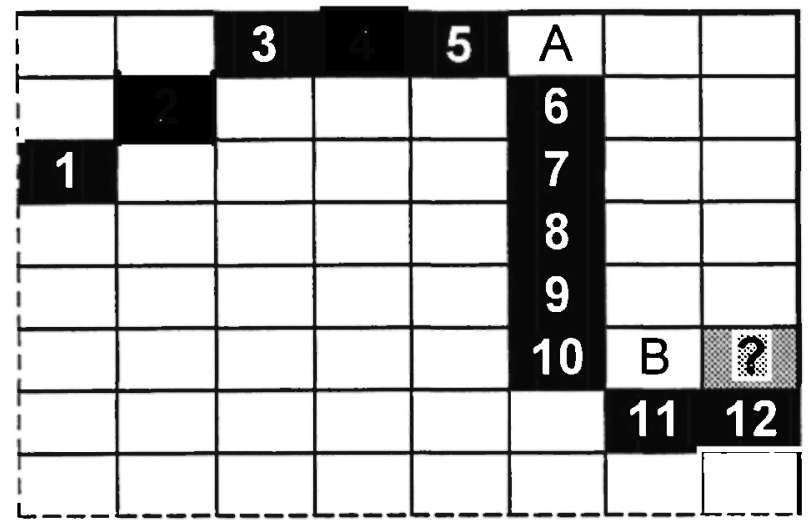

Fig. 8: An example of 'looping'.

\section{EVALUATING THE SOLUTIONS}

Having developed two methods for generating a large number of $8 \times 8$ path-following matrices, there are two reasons for defining some measures of path complexity, and evaluating these measures on samples of matrices produced by these methods. Firstly, in the context of cognitive testing, it is clearly desirable to develop measures of path complexity which may predict the performance of subjects. Secondly, while the tree-search algorithm is clearly faster and more powerful, it embodies the programmer's insights into the appropriateness of the heuristics which serve pruning the search tree, and hence may well incorporate unrecognized constraints. The measures provide a means of comparing the path-following matrices produced by the two methods and, in particular, of ensuring that the paths generated by the tree-search procedure are not idiosyncratic in this sense.

With these two goals in mind, complexity measures based upon the three concepts of moving, turning, and self-intersection were defined and calculated for samples of 64 matrices generated by both the neural network and tree search approaches. The first two concepts, moving and turning, seem instrinsically related to the notion of path-following and may therefore form the basis for many useful measures of path complexity. Meanwhile, the concept of self-intersection is of particular interest since the ZVT is one of the few path-following tests in which it is incorporated. 
Considering firstly the concept of turning, measures were taken of the degree of turn required to make each successive move in a path solution, and the absolute magnitude of this degree of turn. The constrained nature of ZVT paths results in these angular measures falling discretely in multiples of 45 degrees, between 0 and 135 degrees, or between -135 and 135 degrees, depending upon whether or not the absolute value is taken. The average number of turns falling in these categories, for each measure, is shown in Figures 9 and 10.

In addition, for each path, the mean and absolute deviation from this mean of these measures of turn provide further summary statistics of this type of path complexity (Spiegel, 1961). Figures 11 and 12 plot the absolute deviation from the mean against the mean turn, and absolute mean turn, respectively.

Considering the second concept of moving, individual path segment lengths within an overall path solution were defined as being the number of successive moves requiring no turn. It should be noted that this definition equates the path segment length associated with horizontal, vertical and

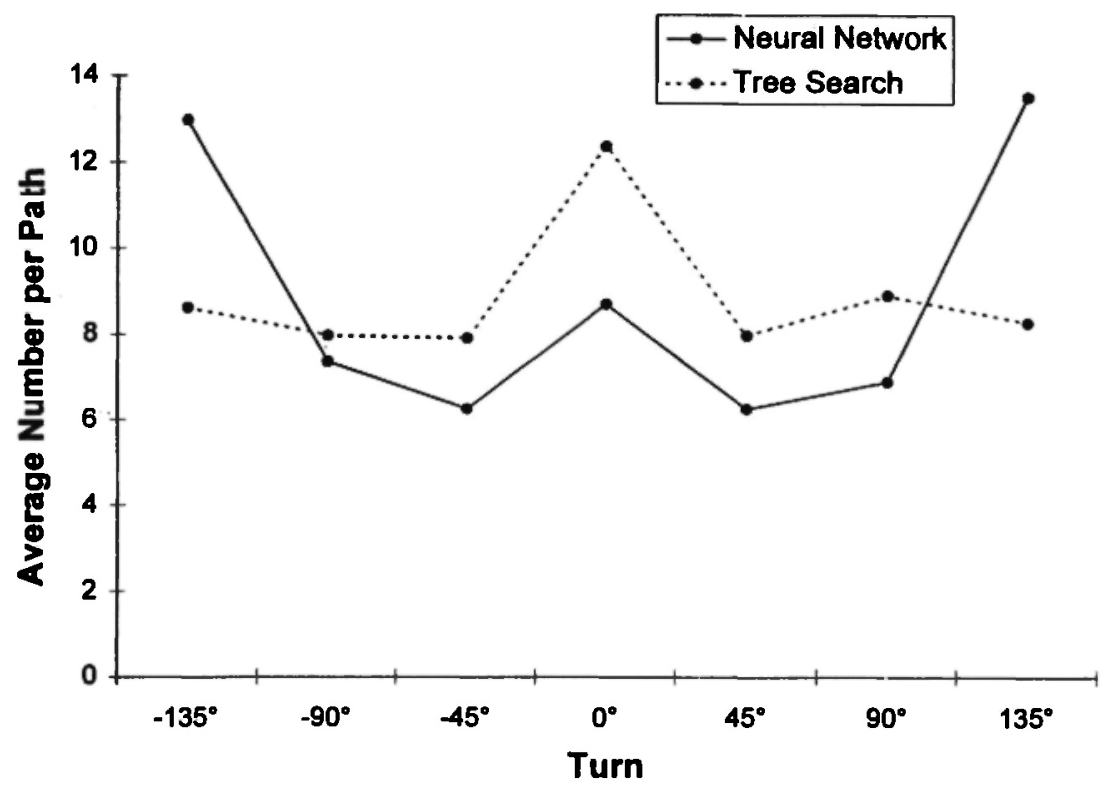

Fig. 9: The distribution of degrees of turn across the two sets of 64 sample matrices, produced by the neural network and tree-search algorithms. 


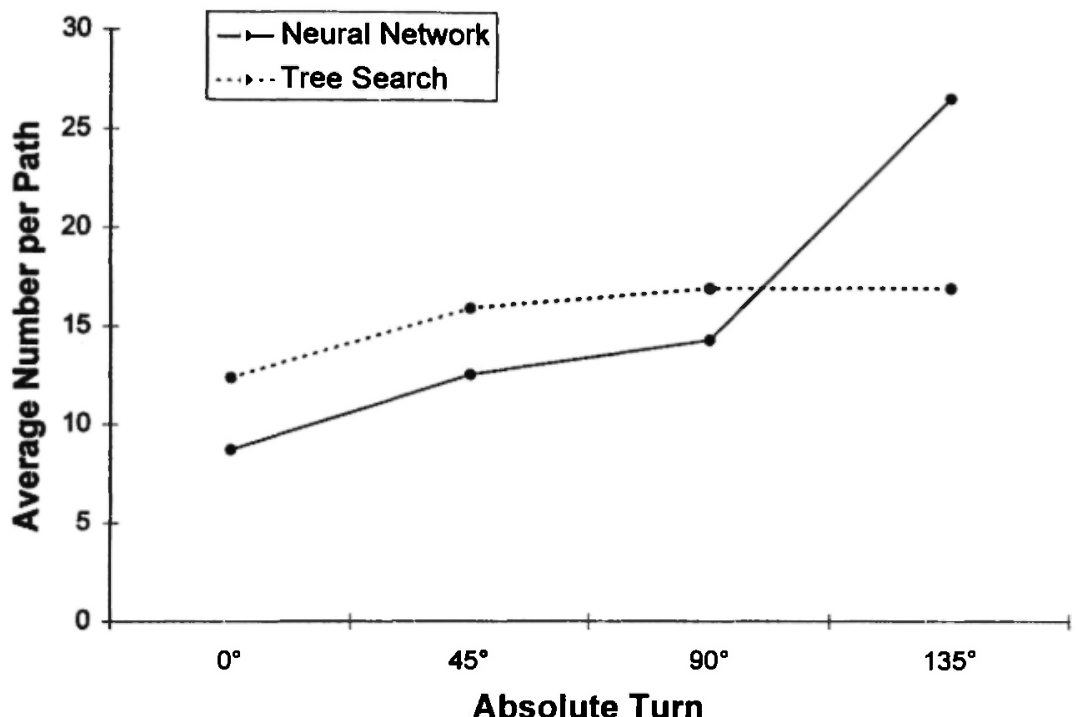

Fig. 10: The distribution of degrees of absolute turn across the two sets of 64 sample matrices, produced by the neural network and treesearch algorithms.

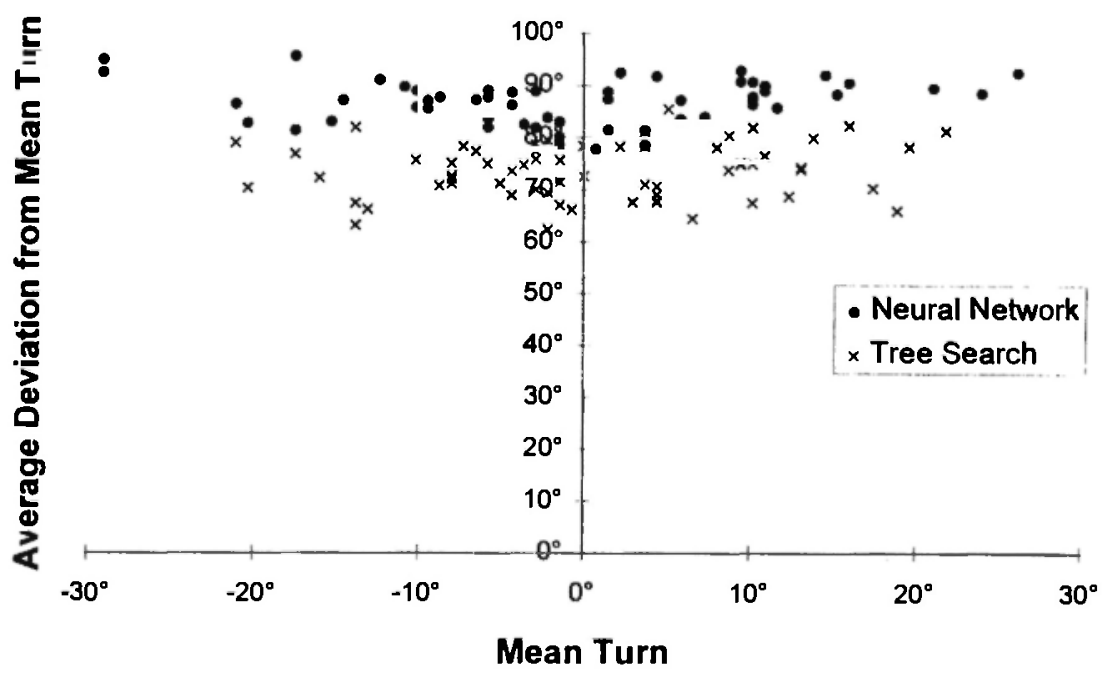

Fig. 11: Average deviation from mean turn plotted against mean turn, for each of the two sets of 64 sample matrices, produced by the neural network and tree-search algorithms. 


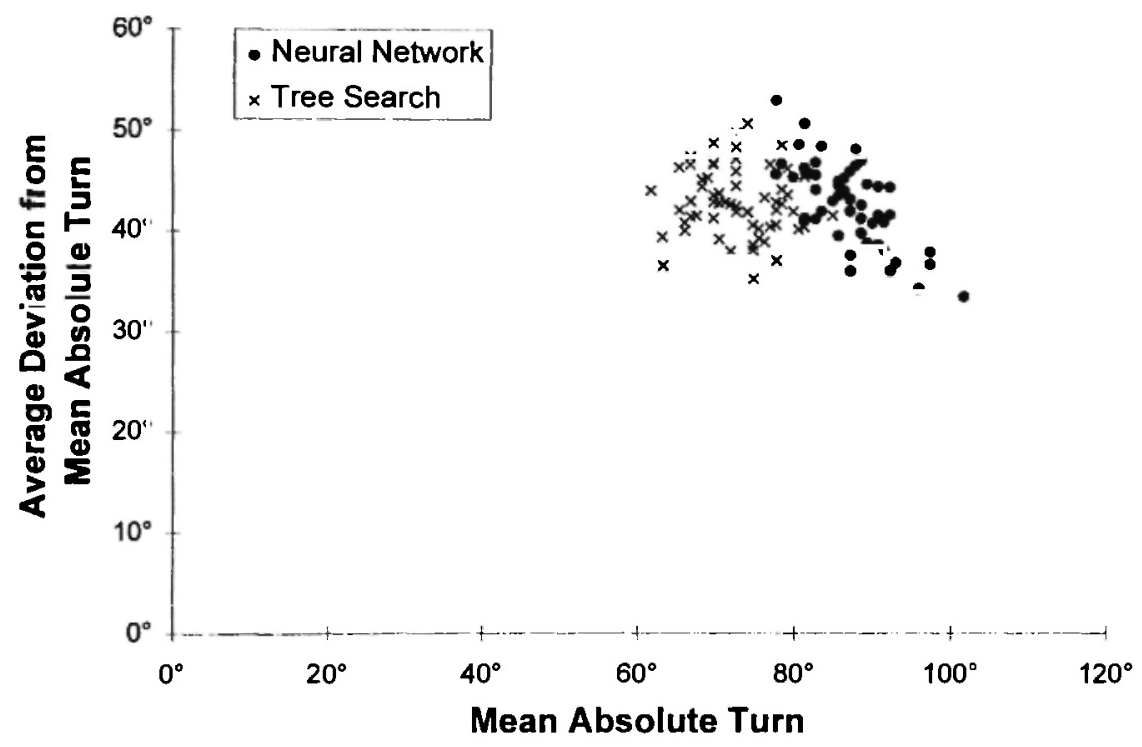

Fig. 12: Average deviation from mean absolute turn plotted against mean absolute turn, for each of the two sets of 64 sample matrices, produced by the neural network and tree-search algorithms.

diagonal movement, and therefore differs from the more intuitive notion of length as Euclidean distance. The average number of path segments of different lengths for the neural network and tree search sample matrices are shown in Figure 13. The mean path length within a solution provides a useful summary statistic, and the results of its calculation are displayed in Figure 14.

Finally, the total number of self-intersections in each of the sample matrices was calculated. Details of the distribution of this measure within the sample matrices are given in Figure 15. As can be seen from Figures 9 to 15 , the paths produced by the neural network and tree-search algorithms are extremely similar. The main difference seems to be that the tree-search algorithm results in slightly less angular variability due to a weaker tendency to make path moves involving a turn through \pm 135 degrees. Given the stochastic nature of both path generation processes, it seems unlikely that this small difference would be detectable by a subject, or would give rise to any measurable effect on performance.

While the main aim of this project was to produce stochastically different ZVT test forms, it is also possible that these techniques may open the way 


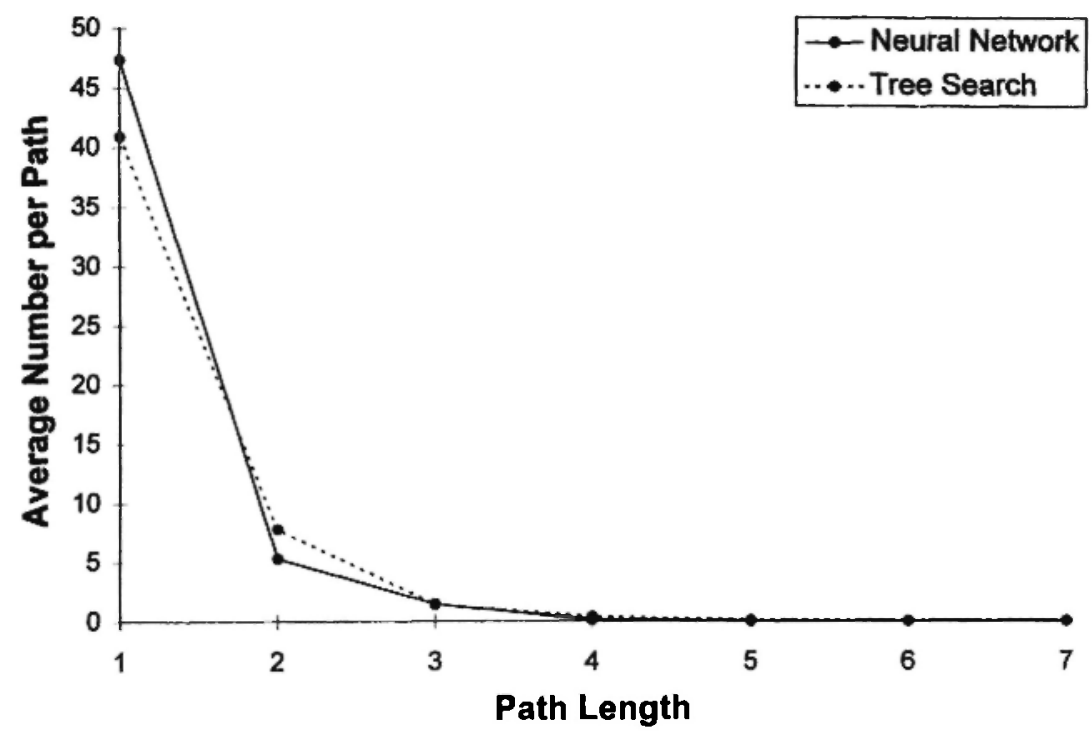

Fig. 13: The distribution of path lengths within the two sets of 64 sample matrices, produced by the neural network and tree-search algorithms.

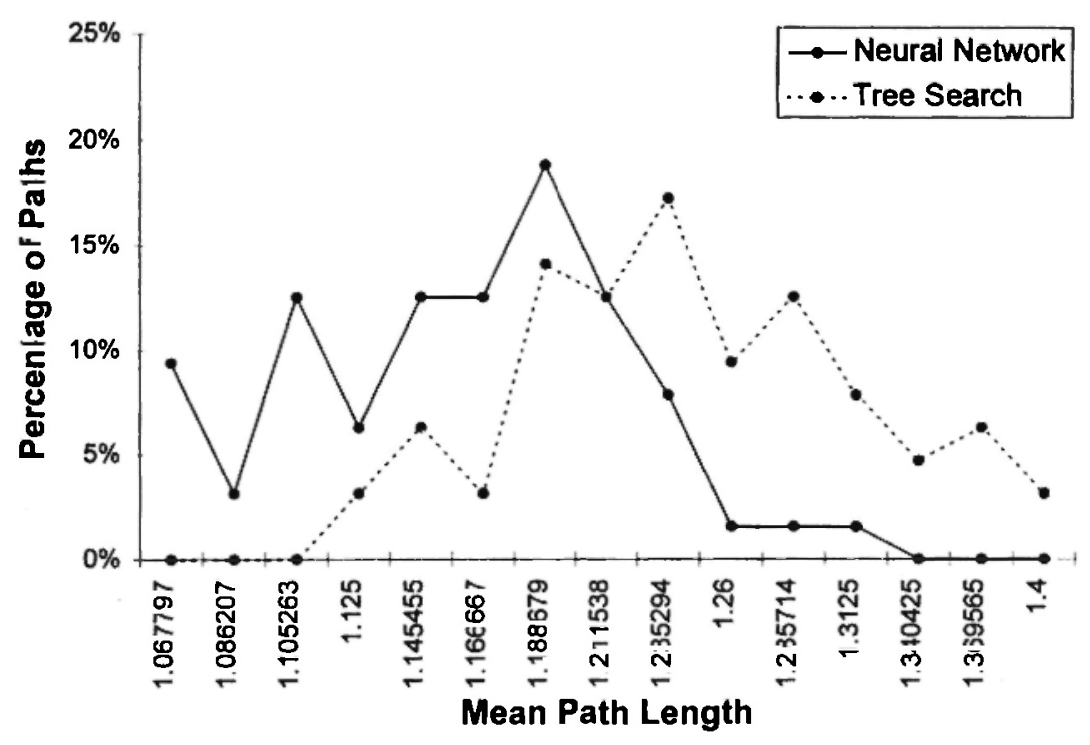

Fig. 14: The distribution of mean path lengths across the two sets of 64 sample matrices, produced by the neural network and tree-search algorithms. 


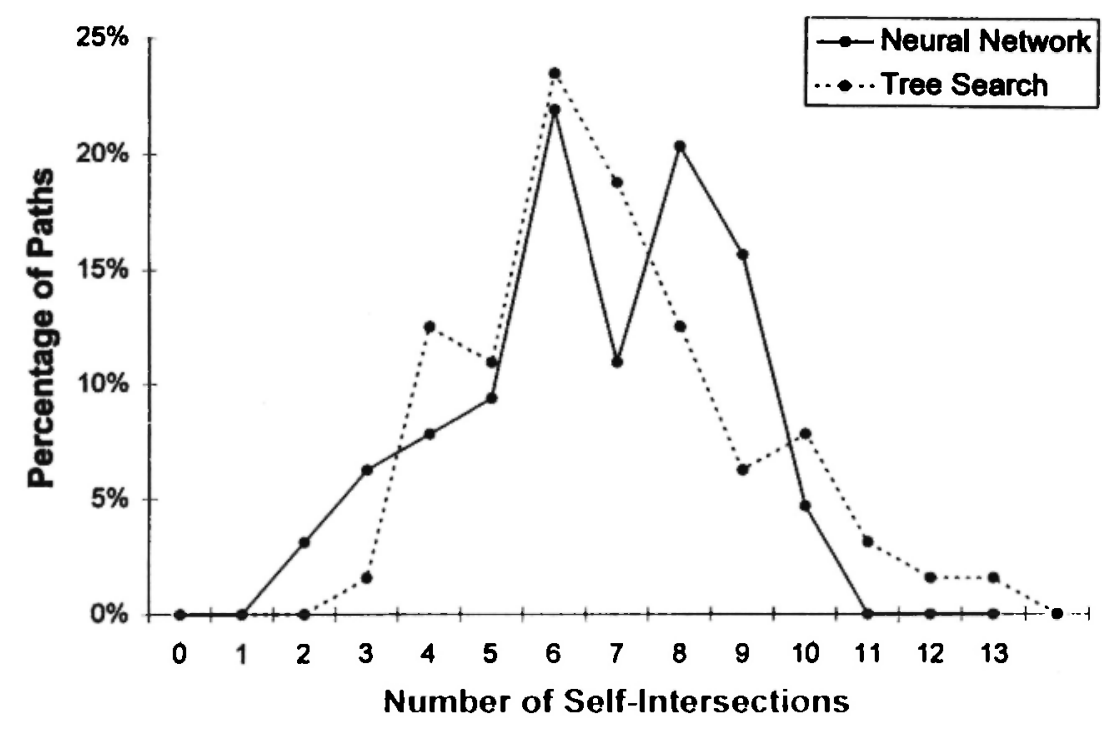

Fig. 15: The distribution of self-intersections across the two sets of 64 sample matrices, produced by the neural network and tree-search algorithms.

for a more systematic study of path following performance. For example, it might prove interesting to examine subjects' sensitivity to constraints or biases in the pathways. Such biases could be introduced by using nonuniform probability distributions or systematic weightings in either the cost matrix of the neural network, or in the random selection of neighboring cells by the tree search algorithm. Since the construction of ZVT pathways can be thought of as the realization of an optimization process, the efficiency with which human subjects can follow such pathways, and adapt to constraints in their generation, seems likely to have more general import for cognitive functioning.

\section{AUTHOR NOTE}

Michael D. Lee, Department of Psychology, University of Adelaide, Adelaide, South Australia; Mark Brown, Department of Psychology, University of Adelaide, South Australia; Douglas Vickers, Psychology Department, University of Adelaide, South Australia. 
The present study is part of an ongoing project on the assessment of cognitive functioning carried out at the Psychology Department, University of Adelaide, in collaboration with the Department of Anaesthesia and Intensive Care at the Royal Adelaide Hospital, Adelaide, South Australia. The study is supported by an Adelaide University Research Scheme Grant to D. Vickers.

Correspondence concerning this article should be addressed to Douglas Vickers, Psychology Department, University of Adelaide, Adelaide, South Australia 5005. Electronic mail may be sent via Internet to Douglas Vickers at psyd-vic@arts.adelaide.edu.au.

\section{REFERENCES}

Ammons, C.H. 1955. Task for the study of perceptual learning and performance variables, Perceptual and Motor Skills, 5, 11-14.

Barnsley, M. 1988a. Fractals Everywhere, New York: Academic Press.

Barnsley, M. 1988b. Fractal modelling of real world images, in: The Science of Fractal Images, edited by Peitgen, H.-O. \& Saupe, D., New York: Springer Verlag, 219-242.

Bord, J. 1976. Mazes and Labyrinths of the World, London: Latimer New Dimensions, Ltd.

Brown, E.C., Casey, A., Fisch, R.I., \& Neuringer, C. 1958. Trail Making Test as a screening device for the detection of brain damage, Journal of Consulting Psychology, 22, 469-474.

Butler, M., Retzlaff, P.D., \& Vanderploeg, R. 1991. Neuropsychological test usage, Professional Psychology: Theory, Research, and Practice, 22, 510-512.

Corrigan, J.D., \& Hinkeldey, N.S. 1987. Relationship between parts A and B of the Trail Making Test, Journal of Clinical Neuropsychology, 43, 402-409.

Crawford, J.R., Parker, D.M., \& McKinlay, W.W. 1992. A Handbook of Neuropsychological Assessment, Hillsdale, NJ: Lawrence Erlbaum.

Durbin, R., \& Willshaw, D. 1987. An analogue approach to the Traveling Salesman problem using an elastic net method, Nature, 326, 689-691.

Elithorn, A. 1955. A preliminary report on a perceptual maze test sensitive to brain damage, Journal of Neurology, Neurosurgery, and Psychiatry, 18, 287-292. 
Elithorn, A., Jones, D., Kerr, M., \& Lee, D. 1964. The effects of the variation of two physical parameters on empirical difficulty in a perceptual maze test, British Journal of Psychology, 55, 31-37.

Elithorn, A., Kerr, M., \& Mott, J. 1960. A group version of a perceptual maze test, British Journal of Psychology, 51, 19-26.

Falkener, E. 1892. Games Ancient and Oriental, London: Longmans.

Fein, S. 1993. First Drawings: Genesis of Visual Thinking, Pleasant Hill, CA: Exelrod Press.

Fritzke, B., \& Wilke, P. 1991. A neural network for the Traveling Salesman problem with linear time and space complexity, International Joint Conference on Neural Networks (Singapore), 929-934.

Geffen, G. 1995. Approaches to neuropsychological assessment: A comment, Australian Psychologist, 30, 45-46.

Gibson, H.G. 1964. The Spiral Maze: A psychomotor test with implications for the study of delinquency, British Journal of Psychology, 55, 219-225.

Gibson, H.G. 1965. Manual to the Gibson Spiral Maze, London: University of London Press.

Goldschlager, L., \& Lister, A. 1988. Computer Science: A Modern Introduction (2nd ed.), New York: Prentice Hall.

Hopfield, J.J., \& Tank, D.W. 1985. Neural computation of decisions in optimization problems, Biological Cybernetics, 52, 141-152.

Lawler, E.L. 1985. The Traveling Salesman Problem: A Guided Tour of Combinatorial Optimization, Chichester, UK: Wiley.

Lezak, M.D. 1995. Neuropsychological Assessment (3rd ed.), New York: Oxford University Press.

Mandelbrot, B. 1982. The Fractal Geometry of Nature, San Francisco: W.H. Freeman \& Co.

Matthews, W.H. 1922. Mazes and Labyrinths, New York: Dover Publications. (Reprinted 1970).

Mehta, S., \& Fulop, L. 1993. An analogue neural network to solve the Hamiltonian Cycle problem, Neural Networks, 6, 869-881.

Murray, H.J.R. 1913. A History of Chess, Oxford: Oxford University Press.

Neubauer, A.C. 1993. Intelligenz und Geschwindigkeit der Informationsverarbeitung: Stand der Forschung und Perspektiven, Psychologische Rundschau, 44, 90-105.

Nevo, B., Arronson, H., \& Israeli, S. 1984. Multiple-choicing the Maze Test, Perceptual and Motor Skills, 58, 923-928.

Newell, A., \& Simon, H.A. 1972. Human Problem Solving, Englewood Cliffs, NJ: Prentice-Hall. 
Oswald, W.D., \& Fleischmann, U.M. 1985. Psychometrics in ageing and dementia: Advances in geropsychological assessments, Archives of Gerontology and Geriatrics, 4, 299-309.

Oswald, W.D., \& Fleischmann, U.M. 1995. Narnberger-Alters-Inventar (NAI), Gottingen, Germany: Hofgrefe.

Oswald, W.D., \& Roth, E. 1978. Der Zahlen-Verbindungs-Test (ZVT), Göttingen, Germany: Hofgrefe.

Oswald, W.D., \& Roth, E. 1987. Der Zahlen-Verbindungs-Test (ZVT): Handanweisung [Manual], Göttingen, Germany: Hofgrefe.

Pearce, C.E.M., \& Vickers, D. 1996. A heuristic for the construction of alternative test forms for the Zahlen-Verbindungs-Test. Perceptual and Motor Skills, 82, 147-152.

Peitgen, H.-O., Jürgens, H., \& Saupe, D. 1992. Chaos and Fractals: New Frontiers of Science, New York: Springer Verlag.

Porteus, S.D. 1965. Porteus Maze Test: Fifty Years' Application, New York: Psychological Corporation.

Reitan, R.M. 1955. The relation of the Trail Making Test to organic brain damage, Journal of Consulting Psychology, 19, 393-394.

Reitan, R.M. 1958. The validity of the Trail Making Test as an indicator of organic brain damage, Perceptual and Motor Skills, 8, 271-276.

Reitan, R.M. 1992. Trail Making Test: Manual for Administration and Scoring, Tucson, AZ: Reitan Neuropsychology Laboratory.

Reitan, R.M., \& Wolfson, D. 1988. Traumatic Brain Injury, Vol. II: Recovery and Rehabilitation, Tucson, AZ: Neuropsychology Press.

Reitan, R.M., \& Wolfson, D. 1992. Neuropsychological Evaluation of Older Children, Tucson, AZ: Neuropsychology Press.

Shepard, R.N. 1974. Representation of structure in similarity data: Problems and prospects, Psychometrika, 39 (4), 373-422.

Spiegel, M.R. 1961. Theory and Problems of Statistics, New York: Schaum.

Spreen, O., \& Strauss, E. 1991. A Compendium of Neuropsychological Tests, Oxford: Oxford University Press.

Tsai, L.S. 1969. A number-joining test for predicting the success of Chinese air cadets, Perceptual and Motor Skills, 29, 623-626.

Vernon, P.A. 1993. Der Zahlen-Verbindungs-Test and other trail-making correlates of general intelligence, Personality and Individual Differences, 14, 35-40.

Vernon, P.A., \& Weese, S.E. 1993. Predicting intelligence with multiple speed of information-processing tests. Personality and Individual Differences, 14, 413-419. 
Vickers, D., Vincent, N., \& Medvedev, A. in press. The geometric structure, construction, and interpretation of path-following (trail-making) tests, Journal of Clinical Psychology.

Voss, R.F. 1988. Fractals in nature: From characterization to simulation. In: The Science of Fractal Images, edited by Peitgen H.-O. \& Saupe, D., New York: Springer Verlag, 21-70.

Walsh, K.W. 1985. Understanding Brain Damage: A Primer of Neuropsychological Evaluation, Edinburgh: Churchill Livingstone. 
1 